A SIMPLE MARKOV MODEL FOR THE ASSESSIENT OF HOST PATCH QUALITY

BY FORAGIKG PARASITOIDS

\author{
by \\ Richard F. Green \\ University of Minnesota, Duluth \\ Toram Ayal
}

The Blaustein Institute for Desert Research

TR $97-1$

Department of Mathematics and Statistics

University of Minnesota, Duluth

Duluth, Minnesota Duluth

January, 1997 


\title{
A SIMPLE MARKOV MODEL FOR THE ASSESSMENT OF HOST PATCH QUALITY BY FORAGING PARASITOIDS
}

\author{
RICHARD F. GREEN
}

Department of Mathematics and Statistics

University of Minnesota

Duluth, Minnesota 55812 U. S. A.

\author{
YORAM AYAL \\ Mitrani Center for Desert Ecology \\ The Blaustein Institute for Desert Research \\ Midreshet Ben-Gurion, 84990 Israel
}


Abstract: Insect parasitoids search for their hosts using a method that may be broken into three parts. First they locate plants which may harbor their hosts, then they assess the quality of these plants to decide whether to search them further for hosts, and finally, if they decide to accept a plant for further search, they exploit the plant by searching for hosts and attacking them when they are found. We study the way that parasitoids assess plant quality by developing a mathematical model based on behavioral observations of foraging parasitoids that attack aphids which infest crucifers. Assessment of plants is based on the concentration of cues produced by hosts that inhabit them. Parasitoids are more likely to exploit plants in which more host cues are detected, and the willingness of a parasitoid to exploit a given plant depends on the quality of other plants that have been visited recently. Plants whose quality exceeds a certain threshold will be accepted for exploitation. The threshold for plant acceptance will change with the experience of the parasitoid, increasing when plants heavily infested with hosts are encountered, decreasing when uninfested plants are encountered. We analyze several rules that might describe how the acceptance threshold changes with parasitoid experience, and for each rule we show how the number of parasitoids willing to accept plants with various levels of infestation depends on the number of plants with various levels of infestation. We then consider different rules for exploitation of hosts on plants and find how the proportion of hosts attacked depends on host density. 


\section{INTRODUCTION}

It is widely believed that the way insect parasitoids search for their hosts has a direct effect on the dynamics of host-parasitoid population interactions. This belief had a place in the assumptions of the early Nicholson and Bailey (1935) model, which explored parasitoid-host dynamics; parasitoid search was assumed to be random within host patches, and parasitoid searching efficiency was expressed by a coefficient called the "area of discovery." The connection between behavior and population dynamics in Nicholson's work and in later work was discussed by Vet (1995) at the Nicholson Centenary meeting. The original Nicholson-Bailey model has been modified by introducing more realistic assumptions about host distribution and parasitoid searching behavior (Hassell 1978). Despite a great deal of work, it is not clear that theorists have found a good explanation of parasitoid-host population dynamics (Murdoch 1994).

Work on parasitoid foraging behavior and parasitoid-host population dynamics has been pursued along several lines, behavioral and ecological, theoretical and empirical, but these lines have not yet converged. The behavioral work has been summarized by Godfray (1994), whose book provides the best, broadest recent review of both theoretical and empirical work on foraging behavior. Optimal foraging theory provides models that show how animals should forage, but these models (Stephens and Krebs 1986) are designed with predators, not parasitoids, in mind, and these models often rely on biologically unrealistic assumptions. Many students of behavior, especially students of insect behavior, have investigated the behavioral mechanisms used by particular species (Bell 1991), but their work usually does not provide 
quantitative descriptions of behavior that can be used in ecological models.

We hope to understand parasitoid foraging behavior and to characterize it well enough to understand its ecological consequences. In this paper, we concentrate on one of the problems faced by a parasitoid: how to choose patches to search for hosts. This problem is related to one of the foraging problems listed by Pyke et al. (1977); namely, what types of patches should be searched. However, instead of asking what types of patches the animal should search, we ask how the animal decides which patches to search. We also ask how the animal exploits a patch once it has been accepted. Once we know what rules an animal uses for patch choice and exploitation we can determine the animal's foraging efficiency.

\section{A conceptual model of parasitoid foraging}

Insect parasitoids search for their hosts over areas that are very large in comparison with themselves, and they often use chemical cues to locate their hosts. We model parasitoid foraging by using a model that resembles the step model that Flanders (1953, following Salt 1938) used to explain how different parasitoid species could specialize on different hosts. Flanders described several steps by which parasitoids select or eliminate their potential hosts. First they find suitable host habitat, then they find potential hosts, then they recognize and accept their hosts, and the hosts that are accepted turn out to be suitable or unsuitable. While Flanders was interested in how parasitoids choose specific hosts, we are interested in how parasitoids exploit the hosts that they choose. We are interested in a different question, but we use the same idea of a foraging process consisting of several steps. In particular, we would like to understand how many hosts would be attacked within a particular host colony or on a particular plant by modeling the behavior that certain parasitoids use to find and attack their hosts. 
We envision three aspects of parasitoid search: (1) search for plants which may be infested by their potential hosts, (2) assessment of plants for the presence of hosts, and if sufficient evidence is detected, (3) acceptance of a plant for further search and exploitation. In this paper we are most interested in plant assessment and exploitation. Recent behavioral studies have suggested that during these steps the parasitoid uses specific cues which first attract it to a respective level and then arrest it at this level while searching for cues at the next level down the hierarchy (Waage 1979, van Alphen and Vet 1986). These studies also indicate that qualitatively different types of cues are used at different levels of the hierarchy. Volatile, longdistance, plant-derived cues guide parasitoids to their host plants, while non-volatile short-distance hostderived cues (that is, kairomones) aid in the detection of hosts (Vet and Dicke 1992). There is some evidence that these two types of cues divide the foraging process into two distinct phases: an early phase during which the parasitoid moves between host patches independently of the number of hosts present in them, and a late phase during which the parasitoid moves and exploits host patches in accordance with host density.

\section{A biological model: the aphid parasitoid Diaeretiella rapae}

Parasitoids assess plant quality by trying to detect cues provided by hosts, such as damage to the plant or chemicals given off by the host. We use parasitic wasps that attack aphids as a model for such behavior. These wasps use honeydew produced by aphids to assess plant quality (Ayal et al. 1996) and as a guide to the location of aphid colonies on a plant (Ayal 1987). The biological basis of our model is provided by the detailed study of the foraging behavior of the aphid parasitoid Diaeretiella rapae. 
The wasp Diaeretiella rapae is the main parasitoid of the cabbage aphid Brevicoryne brassicae, which feeds on crucifer plants both in the wild and in agroecosystems. Diaeretiella is attracted to the aphid food plant by the plant smell (Read et al. 1970), and when on the plant uses aphid honeydew as a kairomone to direct it to the aphid colonies which infest the tips of the inflorescence branches (Ayal 1987). In a behavioral study, Ayal (1987) showed how the parasitoid uses plant architecture and information obtained from honeydew in order to find its aphid hosts. Parasitoids land on and search leaves near the bottom of the plant. If honeydew is detected on the leaves, it must have fallen from aphid colonies above, and the parasitoids fly up to inflorescences at the top of the plant, where aphid colonies may be located. If an inflorescence has no aphids, the parasitoid walks down the stem of the plant looking for honeydew. If honeydew is discovered on the stem, the parasitoid follows the honeydew trail upward until it leads to an aphid colony.

In a recent study, Ayal et al. (1996) found that Diaeretiella uses the amount of honeydew found on the plant leaves to assess plant quality and to decide whether to exploit the plant or reject it. However, the decision whether to explore the inflorescence depends not only on the amount of honeydew found on the current plant but also on the amount of honeydew to which the wasp was exposed on the plant searched previously. Thus, a plant's inflorescences will be explored and exploited if the amount of honeydew on the plant's leaves is higher than that found on the previous plant, but, if the amount of honeydew found is less than that found on the previous plant, the wasp may leave the plant after searching a few leaves without exploring the plant's inflorescences.

It seems that Diaeretiella forms an "opinion" about the quality of the environment based on its experience while foraging. A wasp will have a "good opinion" about the environment if it encounters plants with many 
aphids that produce much honeydew, and it will have a "bad opinion" if it encounters plants with few aphids and little or no honeydew. Shaltiel and Ayal (1995) also studied experimentally what determines how many aphids a wasp attacks within a colony of aphids. Their results showed that wasps use the amount of honeydew that they find as they search along a branch to determine how long to spend exploiting a colony that is found at the end of the branch. When the number of aphids in a colony is fixed, wasps search the colony longer if more honeydew is present on the branch leading to a colony. If the amount of honeydew detected is held constant experimentally, the wasps tend to spend a constant length of time exploiting colonies with different numbers of aphids. When honeydew is held constant, wasps tend to attack a larger number of aphids, but a smaller proportion of aphids, in colonies containing more aphids.

We assume that (a) a parasitoid's decision whether to accept a plant for exploitation or to leave the plant and to resume searching elsewhere is determined by two factors: (i) an "opinion" about the overall quality of the environment, and (ii) the apparent quality of the present plant. A plant will be accepted for exploitation if it seems good compared to the environment as a whole. (b) A parasitoid's opinion about overall environmental quality changes with experience, increasing when a plant with many hosts is encountered, decreasing when a plant with few or no hosts is encountered. (c) The way that a plant is exploited may depend on the information that a parasitoid obtains while assessing the plant.

\section{A MATHEMATICAL MODEL FOR PATCH ASSESSMENT AND EXPLOITATION}

A parasitoid searching for hosts that are distributed in patches must decide whether to exploit patches when they are encountered. We believe that the decision whether or not to exploit a patch depends on the experience that an animal has had in its life and on the information that it has about the quality of 
the present patch. An animal that has found many good patches may be unwilling to exploit a patch that would be exploited by an animal that has found only poor patches. At any given time, a population may consist of animals with different experiences, some willing to exploit only the best patches, while others are willing to exploit almost any patch that is encountered. Which patches are exploited depends on how many patches there are of various types and how many animals are willing to exploit patches of each type.

\section{Willingness to accept patches-the change of opinion about environmental quality}

We are interested in (a) how a parasitoid decides whether or not to exploit a host patch, (b) how the set of patch types that the parasitoid is willing to exploit changes with experience, and (c) how the rules governing patch choice influence the frequency with which various patch types are exploited.

We assume that:

(1) There are various patch types, $X=0,1,2, \ldots, k$, which can be ordered according to quality, with $X=0$ the worst and $X=k$ the best.

(2) These patch types are encountered at random, with constant relative frequencies $P(X=x)=p(x)$ for $x=$ $0,1,2, \ldots, k$. We have considered three cases: a) a "poor environment" has a triangular distribution with more poor patches, $\mathrm{p}(\mathrm{x})=(11-\mathrm{x}) / 55$ for $\mathrm{x}=1,2, \ldots, 10 ; \mathrm{b})$ a "so-so environment" has a uniform distribution, $p(x)=1 / 10$ for $x=1,2, \ldots, 10 ;$ and $c)$ a "good environment" has a triangular distribution with more good patches, $\mathrm{p}(\mathrm{x})=\mathrm{x} / 55$ for $\mathrm{x}=1,2, \ldots, 10$. 
(3) Parasitoids accept patches that meet or exceed some threshold $Y$, which varies according to the experience that the animals have. That is, a parasitoid will exploit a patch of type $\mathrm{X}$ if $\mathrm{X} \geq \mathrm{Y}$. If $\mathrm{X}<\mathrm{Y}$, then the patch will be rejected. This implies that a parasitoid recognizes the quality, $\mathrm{X}$, of the present patch and compares this with the value $\mathrm{Y}$ to decide whether to accept the patch.

(4) We think of the threshold $\mathrm{Y}$ as a parasitoid's "opinion" of overall environmental quality. Each time that a patch is encountered, its quality $\mathrm{X}$ is compared with the opinion $\mathrm{Y}$, the patch is exploited or not, and the parasitoid's opinion $\mathrm{Y}$ changes according to what type of patch was encountered. We assume that the new value of $\mathrm{Y}$, call it $\mathrm{Y}(\mathrm{t}+1)$, depends on the previous value, $\mathrm{Y}(\mathrm{t})$, and on the type of patch encountered, $X(t)$, where time $t$ is measured by the number of patches that have been encountered. That is, we have some function

$$
Y(t+1)=f[X(t), Y(t)]
$$

In general, $\mathrm{Y}$ tends to increase when the parasitoid encounters a patch better than expected [when $\mathrm{X}>\mathrm{Y}$ ], and $\mathrm{Y}$ tends to decrease when the patch is worse than expected [when $\mathrm{X}<\mathrm{Y}$ ].

We have considered four examples of the function $f[X(t), Y(t)]$, which may be thought of as rules for opinion change or learning rules.

(a) "All the way up and all the way down":

$Y(t+1)=X(t)$ 
(b) "One step up and one step down":

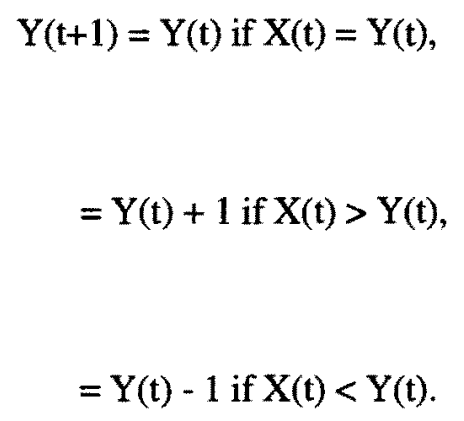

(c) "One step up and all the way down":

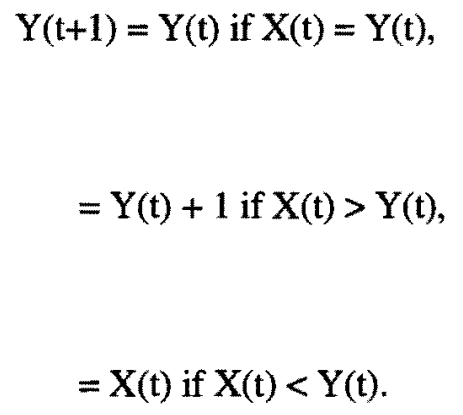

(d) "All the way up and one step down":

$$
\begin{aligned}
& Y(t+1)=Y(t) \text { if } X(t)=Y(t) \\
& =X(t) \text { if } X(t)>Y(t) \\
& =Y(t)-1 \text { if } X(t)<Y(t)
\end{aligned}
$$


Because patch types are assumed to be encountered at random, $Y(t)$ is a Markov process. For each of the rules for opinion change, the matrix of transition probabilities is found by using the rule and the probabilities specifying the patch type frequencies, $\mathrm{p}(\mathrm{x})$. No matter what opinion, $\mathrm{Y}(0)$, that the parasitoid starts with, the distribution of opinions about environmental quality will approach some limiting distribution as more and more patches are visited. We have calculated the limiting distribution by beginning with a parasitoid in a patch of type 1 , that is, $\mathrm{Y}(0)=1$, and iterating through 100 steps. It is not true that all animals will eventually arrive at the same opinion, but after enough time a particular proportion of the animals will have one opinion, a particular proportion will have another one, and so on. These proportions will not change. The distribution of opinions depends on the distribution of patch qualities [the $p(x)$ 's] and the rule that parasitoids use to update their opinions.

\section{Patch acceptance-the probability of accepting a patch}

Once the limiting distribution of opinions, $Y$, has been calculated, it is easy to find the probability that a patch of given quality will be accepted by a random parasitoid. Since we assume that a parasitoid will accept any patch whose quality is at least as high as its opinion, $Y$, of environmental quality, the "probability of accepting" a patch of type $\mathrm{X}$ is just the probability that the parasitoid's opinion will be no higher than $\mathrm{X}$. Thus, the probability that a patch of type $\mathrm{X}$ will be accepted equals the proportion of parasitoids that would accept a patch of that type. That is, we have the probability of accepting a patch of type $x, W(x)=\Sigma g(y)$, where $g(y)$ is the limiting probability that a parasitoid's opinion of environmental quality will be $\mathrm{Y}=\mathrm{y}$. 


\section{Patch exploitation- the probability that a host will be attacked}

None of these calculations require explicit knowledge of the number of hosts in each patch type. For our purposes, "patch type" only refers to information available to parasitoids about patches. We believe that this information depends in some way on the number of hosts present, but knowing the exact relationship between host numbers and a parasitoid's perception about patch quality is not necessary for our model of patch acceptance. It is possible that several different prey densities could lead to the same assessment of patch quality by the parasitoid. That is, a plant with 200 aphids might be as likely to be accepted as a plant with 300 aphids. However, for the sake of simplicity, we assume that each patch of type $x$ contains exactly $N(x)$ hosts. We assume that a parasitoid which decides to exploit a patch of type $x$, containing $N(x)$ hosts, will make an average of $A(x)$ attacks, which depends on the patch type, $x$. The actual number of attacks is assumed to be a random variable having a Poisson distribution with expectation equal to $A(x)$. We assume that within a patch hosts are attacked at random and that a single host may be attacked more than once.

At any time there is some density of parasitoids, which we indicate by $\mathrm{D}$, which is the average number of parasitoids that visit each patch. The average number of parasitoids that decide to exploit a patch of type $\mathrm{x}$ is $L(x)=D^{*} W(x)$, where $W(x)$ is the probability that a patch of type $x$ will be accepted for exploitation. Given particular values of $D, N(x), A(x)$, and given a particular prey distribution, $p(x)$, and a particular rule of updating opinion about the environment, we can calculate the pattern of density dependence that would be observed.

The chance of an individual host in a patch of type $x$ escaping attack by a single parasitoid that exploits the patch is given by 
$\exp [-\mathrm{A}(\mathrm{x}) / \mathrm{N}(\mathrm{x})]$

and if the number of parasitoids that visit each patch is a random variable having a Poisson distribution with mean $=D$, then the expected proportion of hosts in a patch of type $x$ attacked, $M(x)$, is given by

$$
M(x)=1-\exp \{-L(x) *[1-\exp (-A(x) / N(x))]\}
$$

\section{RESULTS}

\section{Distribution of opinion about environmental quality}

In general, at a given time parasitoids will vary in their opinions about environmental quality and therefore in their willingness to accept particular patches for exploitation, depending on their experience. Some individual parasitoids will be willing to exploit poor patches, while others will accept only good ones.

(PUT FIG. 1 HERE.)

a) The limiting distributions for opinion about environmental quality depend on the rules that determine how opinion changes based on experience. A more viscous rule ("one step up and one step down") yields less variable opinion than a less viscous rule ("all the way up and all the way down"). This is illustrated in FIG. 1a, b, c. For each panel of FIG. 1, different curves show the limiting opinions for four different rules for opinion change. The height of each curve represents the proportion of parasitoids that 
would have the opinion that the quality of the environment equals the quality of patch types indicated on the horizontal axis. Asymmetric rules tend to produce higher ("all the way up and one step down") or lower ("one step up and all the way down") opinions than either of the symmetric rules.

b) The distribution of opinion also depends on the overall quality of the environment. Opinion tends to be higher in better environments (FIG. 1c), and more parasitoids would be choosy in good environments. In poor environments (FIG. 1a), parasitoids might be expected to accept almost any patch for exploitation.

\section{The probability of accepting a patch type}

The proportion of parasitoids that are willing to accept patches of a particular type depends on the type. Better patches, that is, patches having more aphids and therefore more honeydew for parasitoids to detect, are more likely to be accepted. The "willingness to accept" patches of particular types is plotted against patch type in FIG. 2 for three different distributions of types, FIG. 2a for a "poor" environment with more patches with fewer hosts, FIG. $2 \mathrm{~b}$ for a "so-so" environment with equal numbers of patches of all types, and FIG. $2 c$ for a "good" environment with more patches with more hosts. For each of the panels, FIG. 2a, b and $c$, curves are given for the same four rules for opinion change that were used in FIG. 1. The curves shown in FIG. 2 show the cumulative distributions corresponding to the opinion distributions shown in FIG. 1. That is, FIG. 1 and FIG. 2 give the same information, but FIG. 2 presents the information in a form more useful in understanding the way that parasitoids choose patch types. The height of the curve in FIG. 2 represents the chance that a parasitoid will accept a patch of a particular type, and a comparison of the heights for different patch types indicates the relative preference for different patch types. 
(PUT FIG. 2 HERE.)

A comparison of the probabilities of accepting patches for different environmental qualities shows that parasitoids will be more discriminating in the good environment, where more good patches are encountered and parasitoids are more likely to have a good opinion about the environment and thus to reject poor patches. When the environment is poor because most patches have few hosts, parasitoids are more likely to have a poor opinion about environmental quality and to accept almost any patch encountered.

In our calculations we have ignored the possibility that patches might contain no hosts at all. A field study conducted at four sites in Israel during 1995, a year with a low density of aphids, showed that only a small fraction of plants harbored aphids (about $10-20 \%$ of plants had aphids). If most plants have no hosts (we might call them "type 0 patches"), then parasitoids might be expected to have a very low opinion of environmental quality and to accept for search and exploitation any plant giving the least indication of the presence of hosts. In this case we might not expect much difference in the chance of acceptance among plants, and any systematic differences that are seen in plant exploitation will be due to the pattern of exploitation and not on the chance of acceptance.

\section{Patch exploitation and density dependence}

Spatial density dependence is illustrated in FIG. 3, which plots the proportion of hosts attacked in a patch, or the mortality rate, $M(x)$, against patch type, $x$, for the same three distributions of patch types as illustrated in FIGs. 1 and 2. In all cases, it is assumed that the number of parasitoids that visit each patch is 
a random variable having a Poisson distribution with a mean, $\mathrm{D}=2$. Some patches will not be visited by any parasitoids, while some will be visited by several. As in FIGs. 1 and 2, the panels in FIG. 3 show what happens for different environments.

\section{(PUT FIG. 3 HERE.)}

In FIG. 3a the environment is poor, more patches are poor, the parasitoid's opinion of the environment is likely to be poor, and most patch types will be accepted for exploitation. On the other hand, in FIG. $3 \mathrm{c}$ the environment is good, more patches are good, the parasitoid's opinion of the environment is likely to be good, and only the better patches are likely to be accepted for exploitation. Thus, the proportion of hosts attacked tends to be quite a bit smaller for poorer patches than for better patches in a good environment, but poorer patches show more nearly the same rate of attack as better patches in a poor environment.

In each panel of FIG. 3, we consider three different patterns of exploitation of patches that are accepted for search. We consider only one rule for opinion change, "all the way up and all the way down." Thus, the curves in each panel of FIG. 3 differ in a different way than the curves in each panel of FIGs. 1 and 2, which each illustrate different rules for opinion change. In all the figures, the horizontal axis represents patch "type," which gives only the order of patch quality, with 10 being best (having the most hosts and most honeydew) and 1 being the worst. However, in order to understand exploitation we must know how many hosts each patch contains, and here we have assumed that the number of hosts in a patch of type $\mathrm{x}$ will be exactly $N(x)=2^{(x-1)}$. Thus, a patch of type $x=5$ will have exactly $2^{(5-1)}=2^{4}=16$ hosts. The three exploitation rules illustrated in each panel of FIG. 3 are: (i) constant number of attacks, (ii) number of attacks proportional to the square root of the number of hosts, and (iii) number of attacks proportional to the 
number of hosts. Parameters are chosen such that the number of attacks would be the same if a patch contained exactly 100 hosts. In this case, the expected number of attacks would equal 20 . When the number of attacks is held constant, the number of attacks $A(x)=20$ for all values of $x$.

In our model there are three factors that affect the pattern of density dependence in patch exploitation seen in FIG. 3.

(a) Since the number of parasitoid visits to each patch is random, some patches will not be visited. Thus, even a patch that would be exploited extensively if visited might not be exploited simply because it happens not to be visited.

(b) In any environment there is at least some preference for better patches. When there are many good patches, this preference may be strong, and a difference in patch exploitation may be due to patch preferences. Thus a patch having few hosts, which could be exhausted by one or a few parasitoids, may be bypassed because it seems poor relative to other patches.

(c) Parasitoids may be limited in the number of eggs that they can lay in a patch (Hafez 1961 has data on egg supply in Diaeretiella rapae). Two of the patterns that we have considered for exploitation (constant number of attacks and number of attacks proportional to the square root of the number of hosts) could be used as models for behavior of egg-limited parasitoids.

The curves shown in FIG. 3 are different according to environmental quality and exploitation rule, but they tend to have in common a lower level of exploitation in patches with low host density, with the rate of 
exploitation rising through intermediate levels of host density and then leveling off or declining for high host density.

The "all the way up and all the way down" rule used in FIG. 3 is appealing esthetically because it is symmetrical and because the limiting distribution of the environmental opinion (FIG. 1) is the same as the distribution of patch types. The "all the way up and one step down" rule may be more realistic biologically. This rule is asymmetrical and tends to keep expectations higher after good patches are encountered, which will lead a parasitoid to concentrate its search in the best patches where the payoff is greatest. Field observations show that a majority of all hosts attacked are attacked in a very small number of patches with the largest number of hosts.

(PUT FIG.4 HERE)

FIG. 4 illustrates patch exploitation for the "all the way up and one step down" rule for opinion change. The cases illustrated in FIG. 4 are the same as in FIG. 3, and the results are roughly similar, with the proportion of hosts attacked increasing with host numbers until the numbers of hosts start to become large, and then leveling off or decreasing in patches with very large numbers of hosts. This leveling off or decrease occurs later (that is, for larger numbers of hosts) in FIG. 4 than in FIG. 3.

\section{DISCUSSION}

In this paper, we have looked at the problem of patch choice, using a model based on some observations of the mechanisms that parasitoids use in deciding whether to accept a patch for search and exploitation. 
Parasitoids of aphids are able to use honeydew produced by their aphid hosts as a measure of patch quality. Experiments have shown that (a) parasitoids are more likely to accept patches in which more honeydew is detected, and (b) the chance of accepting a patch where a given amount of honeydew is detected depends on the quality of patches visited previously (Shaltiel and Ayal 1995, Ayal et al. 1996). We assume that parasitoids choose patches according to their apparent quality, compared with some "opinion" about the overall quality of the environment. We have modeled a parasitoid's change of "opinion" about environmental quality by considering four rules that the parasitoid might use to change opinion based on experience. Given a rule for changing opinion, a rule for accepting patches based on experience and opinion, and the distribution of patch types, it is possible to determine the probabilities that particular patches will be accepted for exploitation. If we also are given a rule for exploiting a patch once it has been accepted, we can determine the pattern of density dependence to expect.

Our approach differs from the approach of optimal foraging theory, which seeks to find the decision that an animal should make. Optimal foraging theory has concentrated on the question of what the best decision is, but not on how the animal might make that decision. The problem of patch choice (which Stephens and Krebs [1986] combined with prey choice under the heading of the "prey problem") is usually formulated in terms of what an animal should do if it knows the quality of the patch being visited, and the overall quality of the environment. The basic question for optimal foraging theorists is not what the animal knows, or how it uses that knowledge to make a decision, but what the decision should be if the animal knew everything. We are not sure what the animal should do, but we have tried to understand what an animal would do, based on some reasonable assumptions about the behavioral mechanisms that the animal uses.

Our results show that animals in the same environment may have different opinions about environmental 
quality because of their different experience, and this means that some animals would be willing to accept a particular patch while others would reject it. That is, the opinion that animals have about the environment is random, taking different values for different individuals. The distribution of opinions depends slightly on what rule we assume that animals use to modify their opinions based on experience, but the distribution of opinion depends strongly on the distribution of patch quality; if most patches contain few hosts, almost any patch will be accepted, while if most patches contain many hosts, only the best patches are likely to be accepted.

When we add patch exploitation to patch acceptance in our model, we see a pattern of spatial density dependence. The pattern of density dependence to be expected depends on the exploitation rule. If a constant number of attacks are made in all patches, the proportion of hosts attacked will be smaller in patches with many hosts, while the proportion can increase with host number if the number of attacks by each parasitoid is proportional to the number of hosts in a patch. It is unlikely that a constant proportion of hosts could be attacked over the entire range of host numbers because egg limitation must restrict attacks in the most densely infested patches.

The pattern of density dependence also depends on the distribution of numbers of hosts in patches. In good environments (in which there are more patches having many hosts), patch acceptance tends to vary more with patch quality than in poor environments and therefore more poor patches will be rejected when the environment is better overall. Quite generally, we would expect the proportion of hosts attacked to increase with host numbers as long as host numbers are small, but the proportion of attacks should tend to level off or decline as the number of hosts becomes large. As can be seen in FIGs. 3 and 4, the exact details depend on environmental quality and the exploitation rule that parasitoids use. The rule that parasitoids use 
to change their opinion about environmental quality can also have a slight effect, as can be seen by comparing FIGs 3 and 4.

It also should be mentioned that the proportion of hosts attacked that is illustrated in FIGs. 3 and 4 refers to the expected proportion attacked. Since the numbers of parasitoids that visit patches are random, the pattern of density dependence that is observed in the field should be expected to show a great deal of variability. Patches with many hosts may happen to be visited by few parasitoids or none, while poorer patches may receive many visits. Field observations in 1995 showed that all plants with more than two aphid colonies were visited and had aphids attacked by at least one parasitoid. This implies that almost all plants received at least a few visits and that the average number of parasitoids visiting each plant was more than one.

Several parts of our model deserve further study. We do not know exactly what rule animals use to change their opinions about environmental quality. Nor do we know exactly what rule animals use to decide whether to search a patch. We do not know whether the exploitation rule that is used once a patch has been accepted depends on previous experience as well as experience in the present patch. We assume that patches of various qualities are encountered at random, but this may not be true. Good patches may be found in clusters, and a parasitoid that finds a plant with many aphids may be likely to find the next plant visited to be heavily infested as well. We also assume that each patch is equally likely to be visited, even though better patches are more likely to be exploited. However, some patches may be easier for parasitoids to find, even though they do not contain more hosts. We only consider the distribution of opinion after a large number of visits, when the distribution of opinion will have become stabilized. If most parasitoids visit only a few patches, then the actual distribution of opinions may be different from the limiting one. 


\section{Other models similar to ours}

There have been three mechanistic models of foraging rules based on the change of opinion with experience, those of Waage (1979), Ollason (1980) and Bernstein et al. (1988). All of this work differs from ours slightly in that it is concerned with patch exploitation, while ours is more concerned with patch assessment.

(1) Our work is closest to that of Bernstein et al. (1988), both in the model that we use and in the consequences of the behavior. They were interested in how predators distribute themselves in patches of prey, in particular, whether predators distribute themselves according to an "ideal free distribution" (Fretwell and Lucas 1970; Fretwell 1972; Sutherland 1983). We are interested in how parasitoid exploitation of hosts is related to host density; in particular, whether parasitoids show density dependent aggregation to host colonies.

Bernstein et al. (1988) modeled the change of a predator's opinion about the overall quality of the environment by using the linear operator model that had been used by Bush and Mosteller $(1951,1955)$ to model response preference in mathematical psychology. Bernstein et al. broke time into discrete intervals, and after each interval each predator was assumed to modify its opinion of the environment by using a weighted average of its previous opinion and the experience that it had had during the latest interval in the present patch. Predators were assumed to have perfect knowledge of the rate of finding prey (the rate 
achieved by all foragers in the patch) at the present time, and this known rate was compared with each animal's opinion. Animals whose opinion of overall environmental quality was higher than the rate of finding prey being achieved in the present patch would leave and choose another patch at random, while predators with lower opinions of the environment would remain in the present patch. Bernstein et al. concluded that under some conditions, especially if prey were not depleted, or if they were depleted slowly, the pattern of aggregation of predators in prey patches might approach an ideal free distribution.

(2) Waage (1979) proposed a behavioral mechanism that produced a rule that parasitoids might use to decide when to leave host patches. Parasitoids search patches for hosts, and eventually leave from the edge of the patches. Waage hypothesized that a parasitoid would begin its search in a patch with some knowledge about the number of hosts present in the patch. The parasitoid was assumed to begin its search with some level of "responsiveness," which is the tendency to respond to a patch edge by turning inward and resuming search. Responsiveness was assumed to decrease continuously between encounters with hosts, but each time that a host was encountered, the level of responsiveness was increased. Parasitoids would leave a patch after their levels of responsiveness fell below some critical threshold.

Stephens and Krebs (1986) pointed out that mechanisms similar to that proposed by Waage (1979) could produce other patch-leaving rules. Theorists have sometimes been misled by the difficulty that they had finding optimal foraging strategies into believing that it would be just as difficult for an animal to implement the strategies. What mechanistic models such as Waage's show is that it may be very easy for an animal to use a strategy that is optimal, or almost optimal. Recent empirical work has studied details about the rules that parasitoids use to leave host patches (Haccou et al. 1991; Hemerik et al. 1993). This work gives a statistical description of some aspects of foraging behavior, but it would be useful if the description could be 
conveniently summarized so that the behavior could be modeled as nicely as it can by using hypothetical behavioral mechanisms such as that proposed by Waage.

(3) Ollason (1980) proposed a hydraulic model to explain how a forager might implement the behavior required by Charnov's (1976) "marginal value theorem." Ollason represented the "amount of food that an animal remembers eating" as the level of fluid in a reservoir. The fluid would flow in as food was eaten, and it would flow out at a rate proportional to the level of the fluid in the reservoir. A forager would remain in a patch as long as its feeding rate was higher than the remembered level, that is, as long as the level of fluid in the reservoir was rising. If travel time were longer, the fluid level would reach a lower level during travel and the fluid would have further to rise, leading to longer stays in each patch. If patches contained more prey, the fluid would rise higher during each patch visit and would be higher at the beginning of the visit to the next patch. Thus, the time to reach the leaving threshold in the next patch would be less. Increasing travel time would increase patch residence time, while increasing patch quality would decrease residence time in patches of fixed quality, just as predicted by Charnov's marginal value theorem.

The point of Ollason's paper was to criticize Charnov's (1976) optimal foraging approach and to suggest a mechanistic alternative to Charnov's marginal value theorem. Ollason's criticism of optimal foraging theory was extended (Pierce and Ollason 1987) in an exchange with Stearns and Schmid-Hempel (1987). We have no quarrel with optimal foraging theory on philosophical grounds. Mechanistic models and optimal foraging theory are not incompatible. In fact, the solution of an optimal foraging problem is sometimes a simple rule that could result from a simple behavioral mechanism (Green 1984, 1987). Optimal foraging theorists have tended to ignore the behavioral mechanisms necessary to implement the solutions to their models. However, optimal foraging theory has the advantage that it requires theorists to 
think about and describe the problems that animal face. When quantitative models are used, optimal foraging theory also permits the quantitative comparison of solutions that animals might use to solve the problems that they face.

Despite the advantages of optimal foraging theory, we have not used an optimization model because we cannot give a simple description of the problems that a foraging parasitoid faces. Instead, we have proposed a simple, mechanistic model based on experimental studies of parasitoid behavior. We have looked at only part of the problem that a parasitoid faces, and even so, our quantitative assumptions have outrun the data. One value of a quantitative treatment, even when not strictly justified by observations, is that it tells which assumptions are important and what facts are necessary for a more accurate and refined model.

\section{CONCLUSION}

The purpose of foraging models is to help understand foraging behavior. One of our main goals is to characterize foraging behavior, and to do so in a way that is useful in understanding the consequences of foraging. Ecology can tell us what problems a forager faces. Behavioral studies can tell us how the forager solves the problems. Optimal foraging theory can tell us how good the solution is. Mechanistic models can tell us what the ecological consequences of foraging are. We have tried to show how parasitoid assessment of host plant quality can influence which plants are searched, and how host distribution, assessment strategy and exploitation strategy can affect the distribution of attacks among hosts. 


\section{ACKNOWLEDGEMENTS}

This study was supported by Grant No. 91-00186 of the United States-Israel Binational Science Foundation (BSF) to Y. Ayal and R. F. Green. 


\section{LITERATURE CITED}

van Alphen, J. J. M., and Vet, L. E. M. 1986. An evolutionary approach to host finding and selection. In: Insect Parasitoids, edited by J.K. Waage and D. Greathead, Academic Press, London, pp. 23-61.

Ayal, Y. 1987. The foraging strategy of Diaeretiella rapae McIntosh. I. The concept of the elementary unit of foraging. Journal of Animal Ecology 56:1057-1068.

Ayal, Y, Shaltiel, L, and Green, R. F. 1996. The use of a kairomone for foraging decisions at two hierarchical scales by an aphid parasitoid: foraging at the habitat level and patch identification. Manuscript.

Bell, W. J. 1991. Searching Behavior. Chapman and Hall, London.

Bernstein, C., Kacelnik, A., and Krebs, J. R. 1988. Individual decisions and the distribution of predators in a patchy environment. Journal of Animal Ecology 57:1007-1026.

Bush, R. R., and Mosteller, F. 1951. A mathematical model for simple learning. Psychological Review $58: 313-323$.

Bush, R. R., and Mosteller, F. 1955. Stochastic Models of Learning. John Wiley, New York.

Charnov, E. L. 1976. Optimal foraging: the marginal value theorem. Theoretical Population Biology 9:129- 
136.

Flanders, S. E. 1953. Variations in susceptibility of citrus-infecting coccids to parasitization. Journal of Economic Entomology 46:266-269.

Fretwell, S. D. 1972. Populations in a seasonal environment. Princeton University Press, Princeton, New Jersey.

Fretwell, S. D., and Lucas, H. J. Jr. 1970. On territorial behavior and other factors influencing habitat distribution in birds. Acta Biotheoretica 19:16-36.

Godfray, H. C. J. 1994. Parasitoids. Princeton University Press, Princeton, New Jersey.

Green, R. F. 1984. Stopping rules for optimal foragers. American Naturalist 123:244-256.

Green, R. F. 1987. Stochastic models of optimal foraging. In: Foraging Behavior, edited by A. C. Kamil, J. R. Krebs, and H. R. Pulliam, Plenum Press, New York, pp. 273-302

Haccou, P., de Vlas, S. J., van Alphen, J. J. M., and Visser, M. E. 1991. Information processing by foragers: effects of intra-patch experience on the leaving tendency of Leptopilina heterotoma. Journal of Animal Ecology 60:93-106.

Hafez, M. 1961. Seasonal fluctuations in population density of the cabbage aphid Brevicoryne brassicae (L.) 
in the Netherlands, and the role of its parasitoid Aphidius (Diaerietiella) rapae (Curtis). Tijdschrift over Plantzenziekten 67:445-548.

Hassell, M. P. 1978. The dynamics of arthropod predator-prey systems. Princeton University Press, Princeton, New Jersey.

Hemerik, 1., Driessen, G., and Haccou, P. 1993. Effects of intra-patch experiences on patch time, search time and searching efficiency of the parasitoid Leptopilina clavipes. Journal of Animal Ecology 62:33-44.

Murdoch, W. W. 1994. Population regulation in theory and practice. Ecology 75:271-287.

Nicholson, A. J., and Bailey, V. A. 1935. The balance of animal populations. Part 1. Proceedings of the Zoological Society of London 3:551-598.

Ollason, J. G. 1980. Learning to forage--optimally? Theoretical Population Biology 18:44-56.

Pierce, G. J., and Ollason, J. G. 1987. Eight reasons why optimal foraging theory is a complete waste of time. Oikos 49:111-118.

Pyke, G. H, Pulliam, H. R., and Charnov, E. L. 1977. Optimal foraging: a selective review of theory and tests. Quarterly Review of Biology 52:137-154.

Read, D. P., Feeny, P. P., and Root, R. B. 1970. Habitat selection by the aphid parasite Diaeretiella rapae. 
(Hymenoptera: Braconidae) and hyperparasite, Charips brassicae (Hymenoptera: Cynipidae). Canadian Entomologist 102:1567-1578.

Salt, G. 1938. Experimental studies in insect parasitism. VI. Host suitability. Bulletin of Entomological Research 29:223-246.

Shaltiel, L., and Ayal, Y. 1995. The role of host-associated and host-derived cues in the foraging decisions of a parasitoid--a case study of patch perception in foragers. Manuscript.

Stearns, S. C., and Schmid-Hempel, P. 1987. Evolutionary insights should not be wasted. Oikos 49:118-125.

Stephens, D. W., and Krebs, J. R. 1986. Foraging Behavior. Princeton University Press, Princeton, New Jersey.

Sutherland, W. S. 1983. Aggregation and the 'ideal free' distribution. Journal of Animal Ecology 52:821828.

Vet, L. E. M. 1995. Parasitoid foraging: the importance of variation in individual behaviour for population dynamics. Paper delivered at "The Nicholson Centenary Meeting: Frontiers of population ecology," 18-22 April 1995, Canberra, Australia.

Vet, L. E. M., and Dicke, M. 1992. Ecology of infochemical use by natural enemies in a tritrophic context. Annual Review of Entomology 37:141-172. 
Waage, J. K. 1979. Foraging for patchily-distributed hosts by the parasitoid Nemeritis canescens. Journal of Animal Ecology 48:353-371. 


\section{FIGURE LEGENDS}

FIG. 1. Parasitoid opinion of environmental quality. A parasitoid holding opinion $\mathrm{x}$ about environmental quality would be willing to accept any patch at least as good as patch type $\mathrm{x}$. Opinion frequency is the proportion of parasitoids holding a certain opinion after many patches have been visited. Panels $a, b$ and $c$ correspond to poor, so-so and good environments, respectively. Diamonds represent the "one step up and one step down" rule for opinion change, squares represent the "one step up and all the way down" rule, triangles the "all the way up and one step down" rule, and crosses the "all the way up and all the way down" rule. The distribution of patch types in the environment is the same as the opinion distribution for the "all the way up and all the way down" rule, indicated by crosses.

FIG. 2. Parasitoid willingness to accept patches. The proportion of parasitoids willing to accept for search and exploitation patches of particular types. Panels $\mathrm{a}, \mathrm{b}$ and $\mathrm{c}$ represent poor, so-so and good environments, respectively. Within each panel, curves represent opinion-change rules as in FIG. 1.

FIG. 3. Expected proportion of hosts attacked in various patch types. Panels $\mathrm{a}, \mathrm{b}$ and $\mathrm{c}$ represent poor, so-so and good environments, respectively. It is assumed that the average number of parasitoid visits per patch is two. Three patterns of patch exploitation are considered. Each would yield the same degree of exploitation if patches contained 100 hosts--which would fall on the patch-type axis somewhere between types 7 and 8 . This is the point for which the curves cross. Diamonds indicate that a constant expected number of hosts $(=$ 20) are attacked in each patch type by each parasitoid that decides to exploit the patch. Squares indicate that the number of hosts attacked is proportional to the square root of the number of hosts present, and triangles indicate that the number of hosts attacked is proportional to the number of hosts present. In each case it is assumed that the parasitoid uses the "all the way up and all the way down" rule to change opinion. 
FIG. 4. Expected proportion of hosts attacked in various patch types. FIG. 4 is the same as FIG. 3 except that in FIG. 4 it is assumed that the parasitoid uses the "all the way up and one step down" rule to change opinion about environmental quality. 
Figure 1a. Distribution of opinion for a poor environment

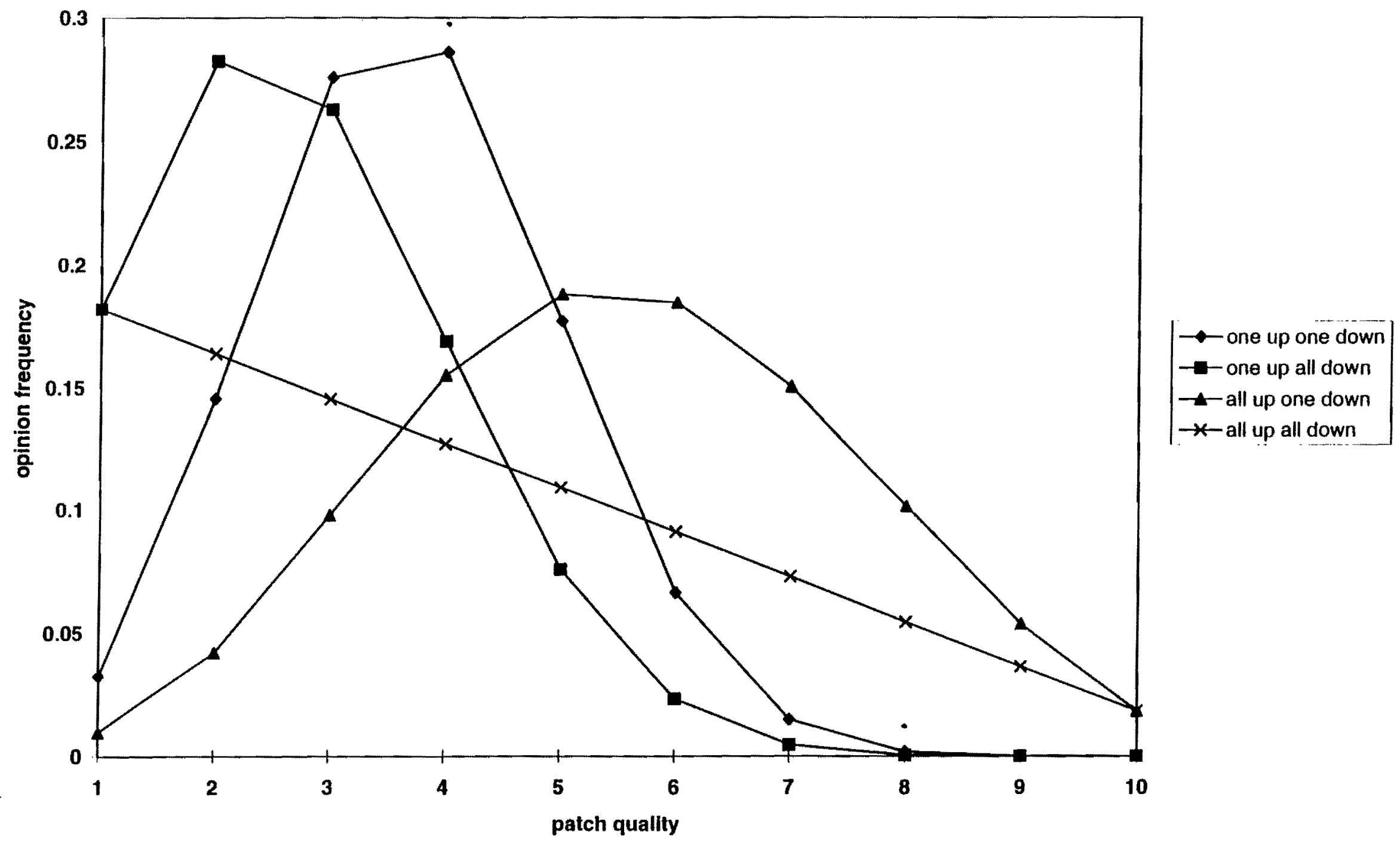


Figure 1b. Distribution of opinion for a so-so environment

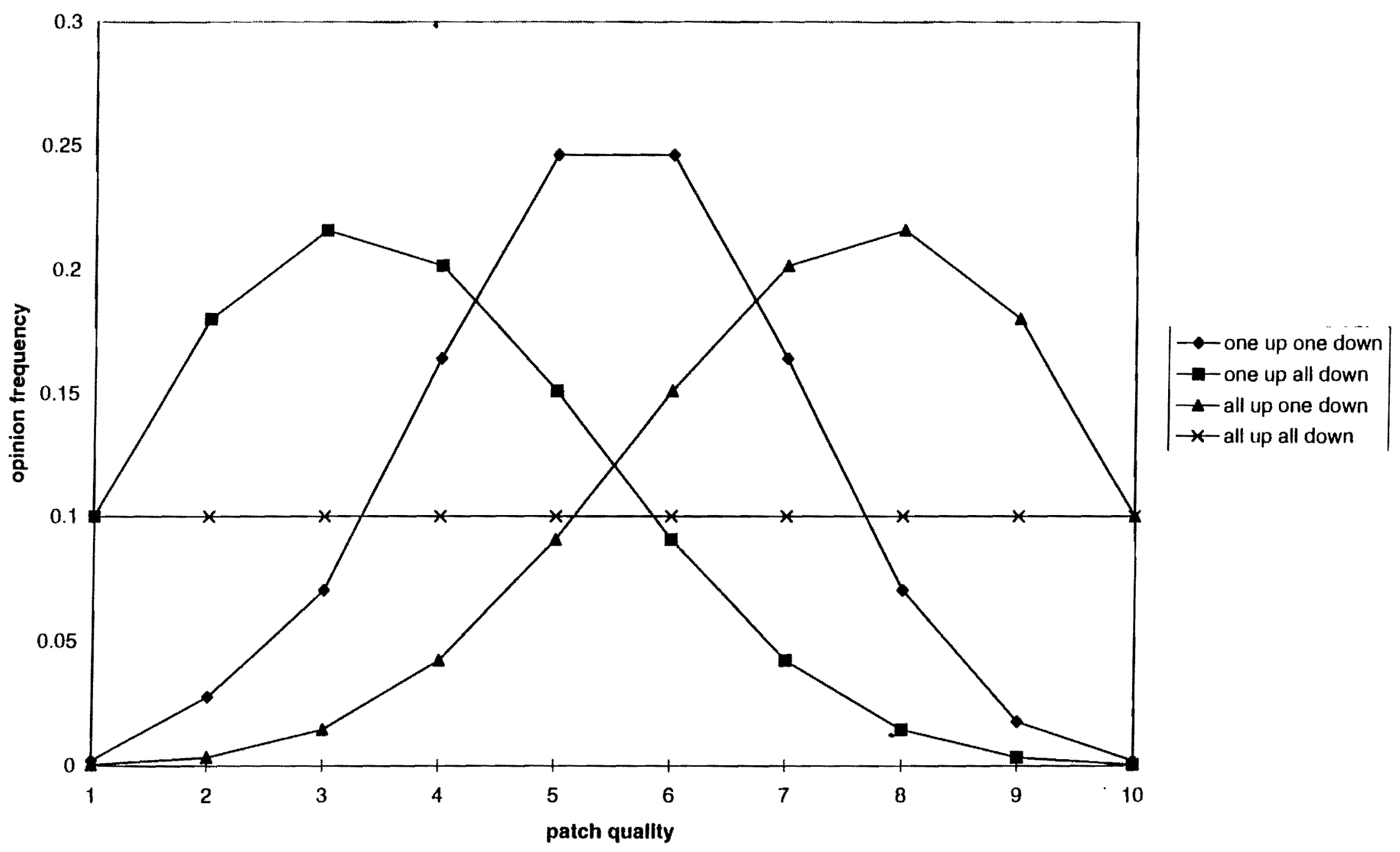


Figure 1c. Distribution of opinion in a good environment

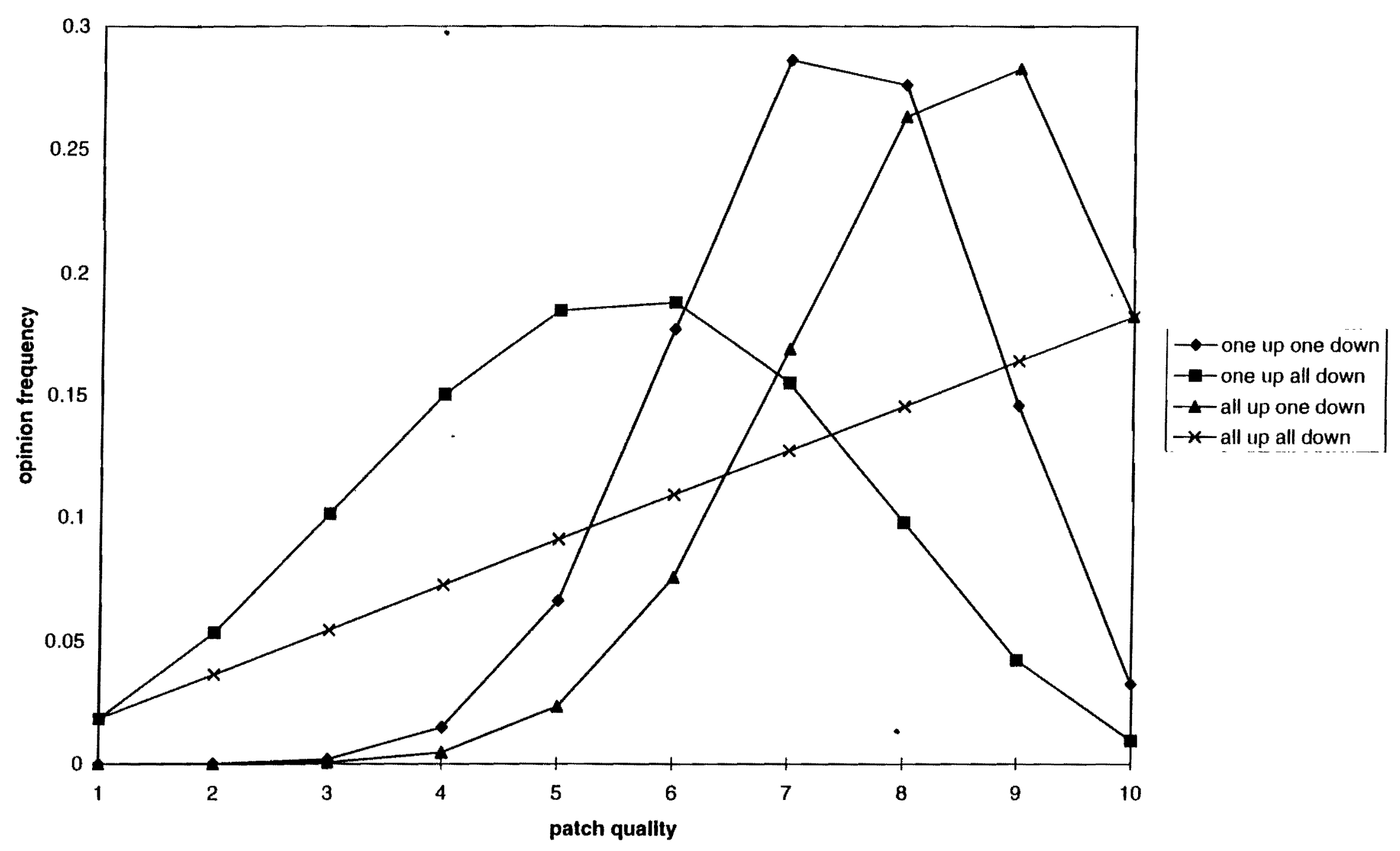


Figure 2a. Willingness to accept patches in a poor environment

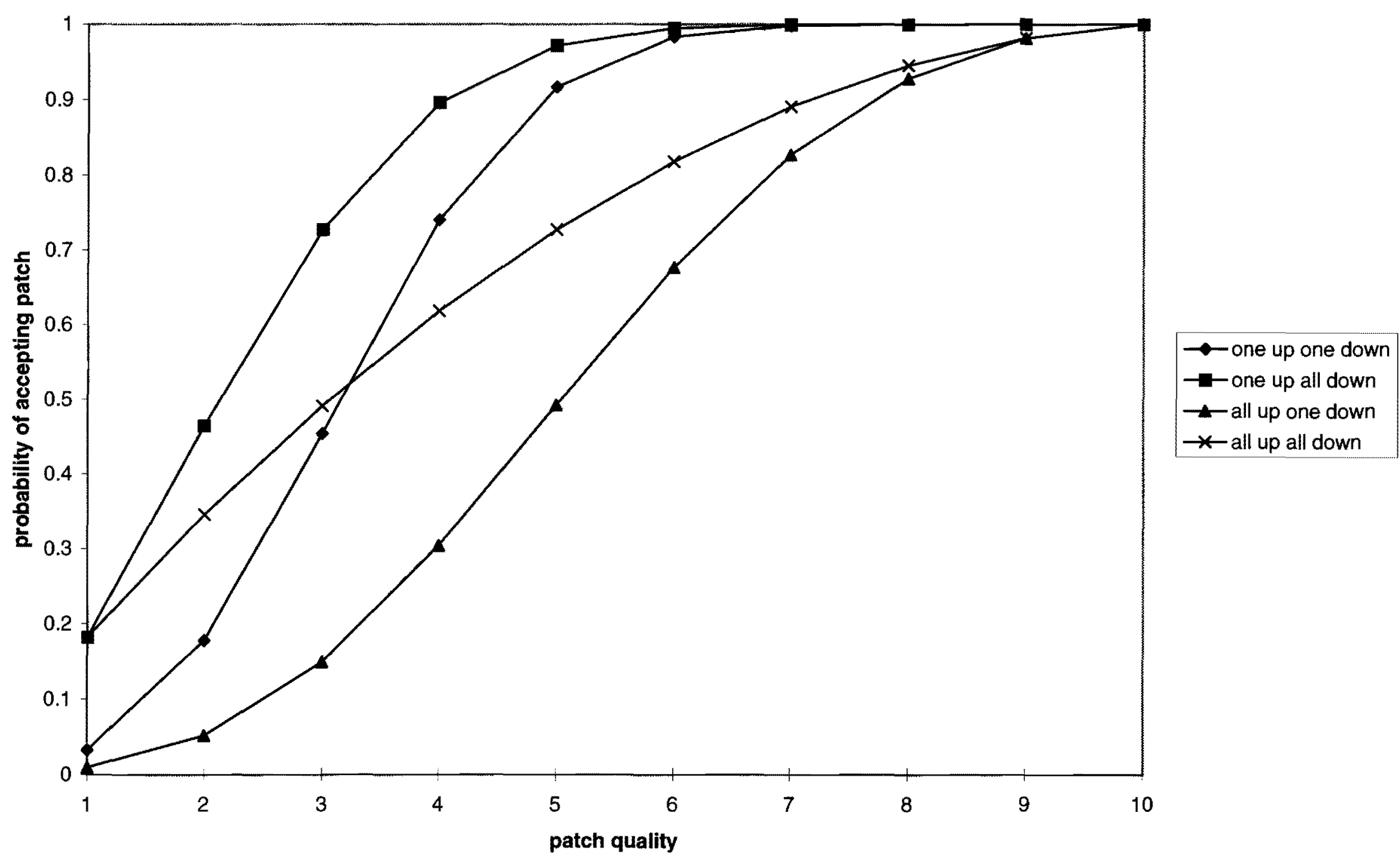


Figure $2 b$. Willingness to accept patches in a so-so environment

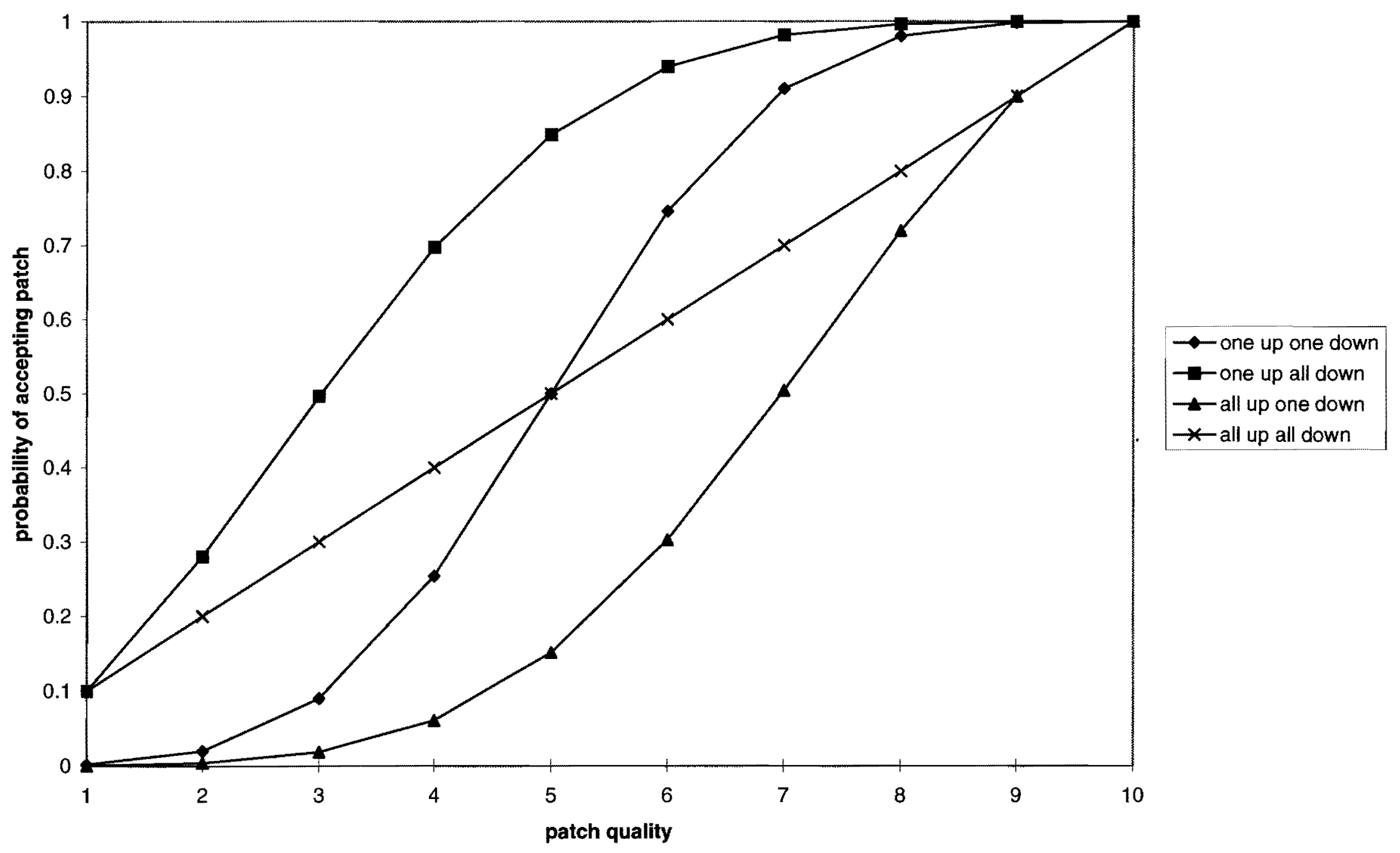


Figure 2c. Willingness to accept patches in a good environment

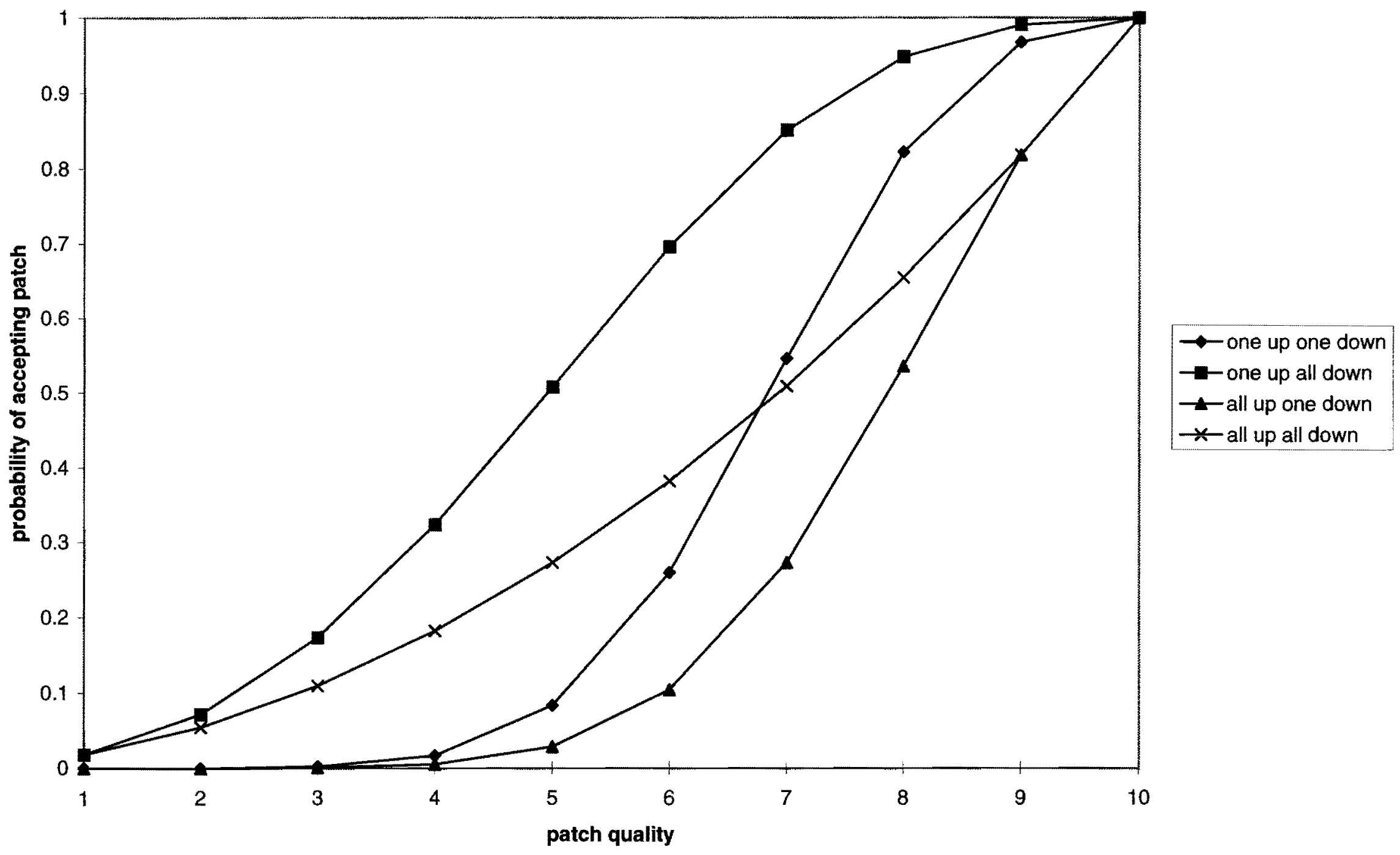


Figure 3a. Proportion of hosts attacked in a poor environment

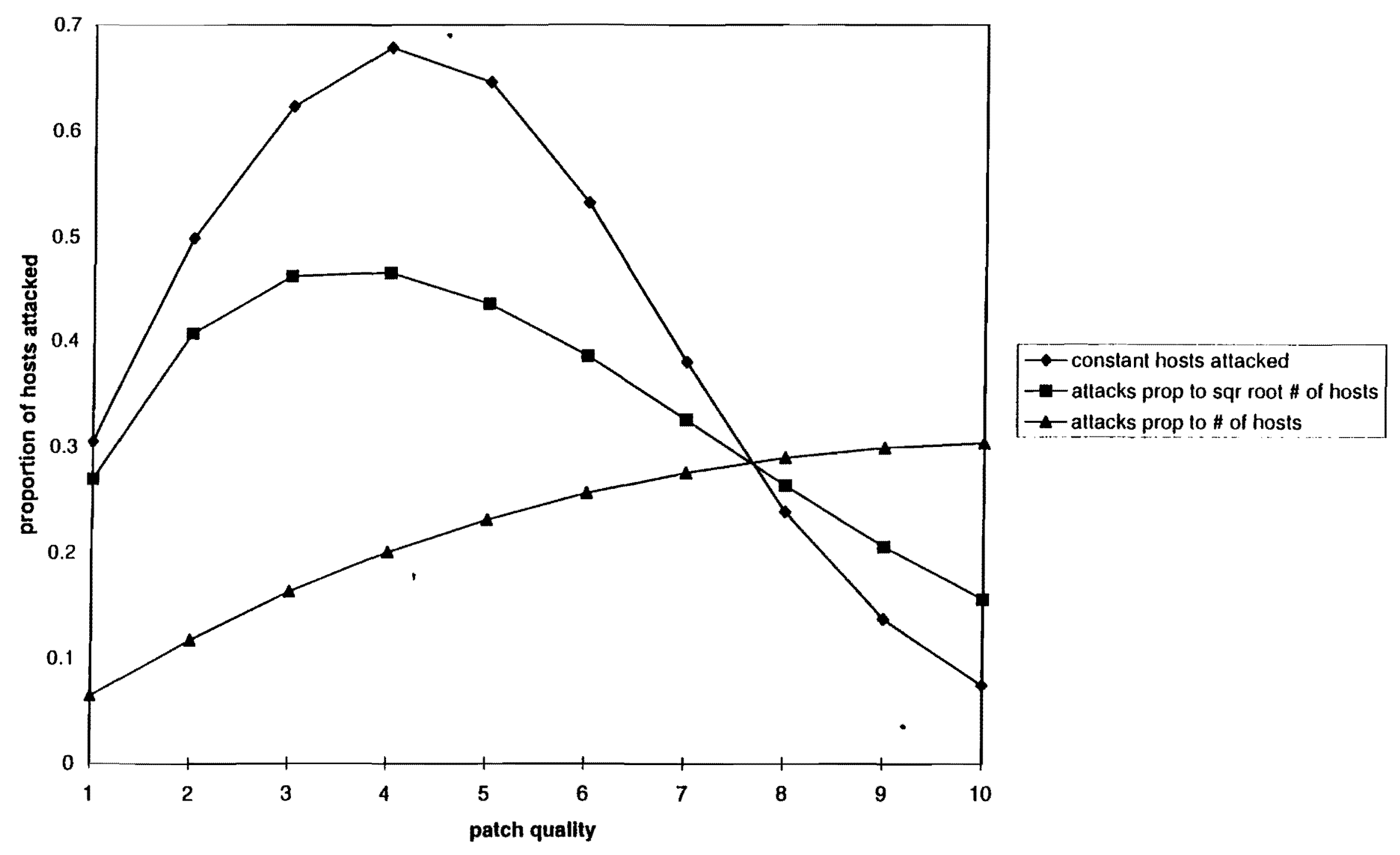


Figure 3b. Proportion of hosts attacked in a so-so environment

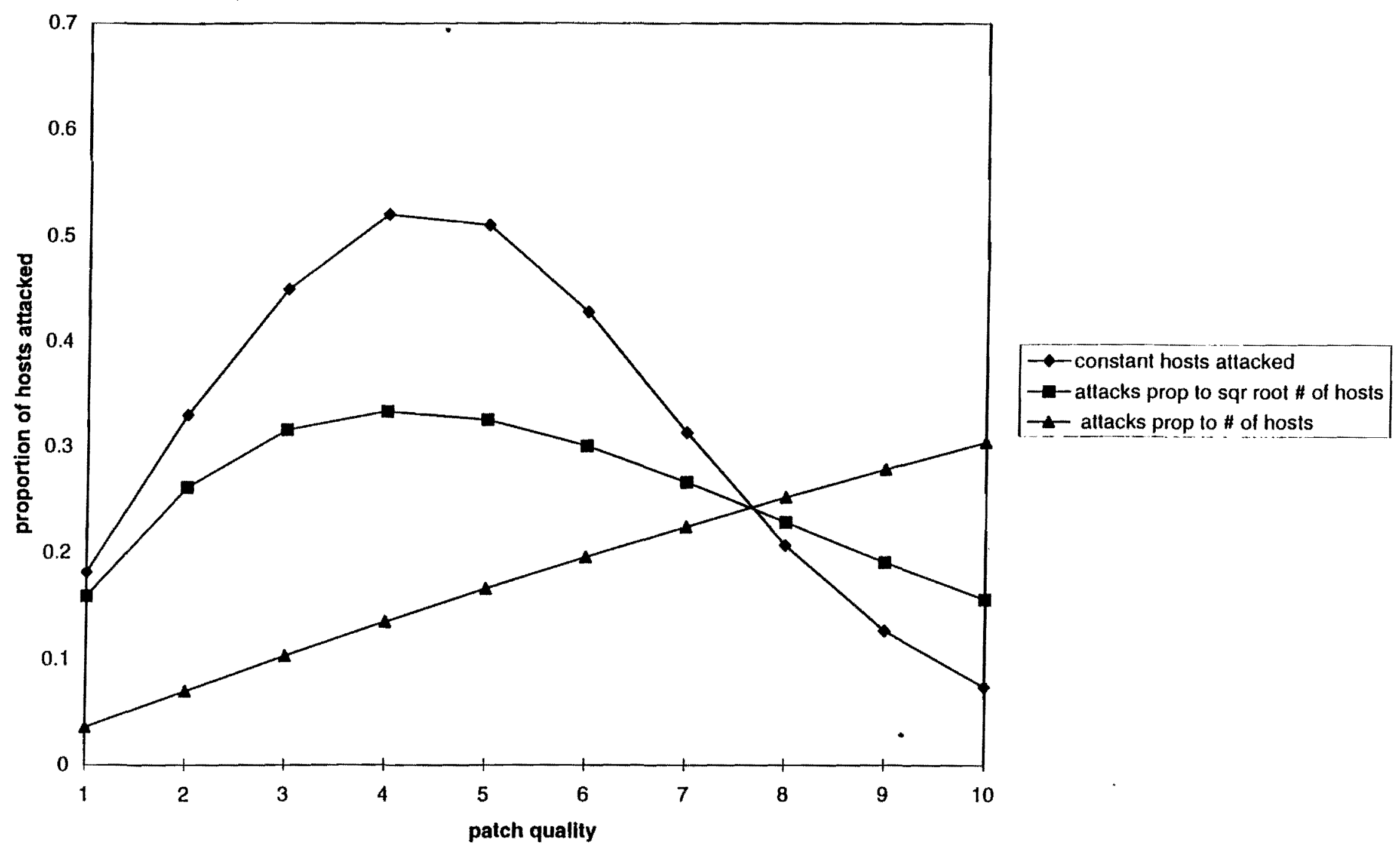


Figure 3c. Proportion of hosts attacked in a good environment

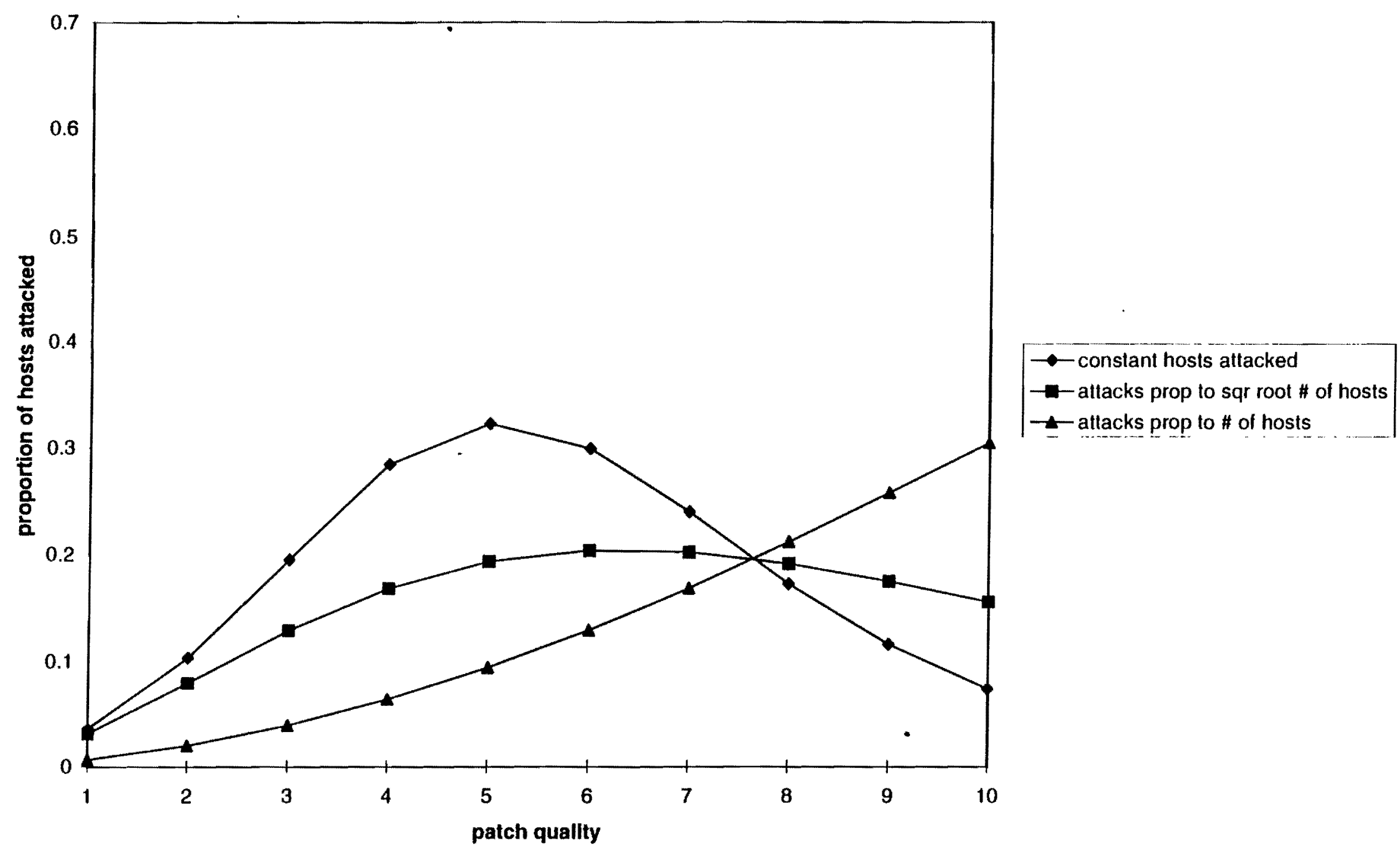


Figure 4a. Proportion of hosts attacked in a poor environment

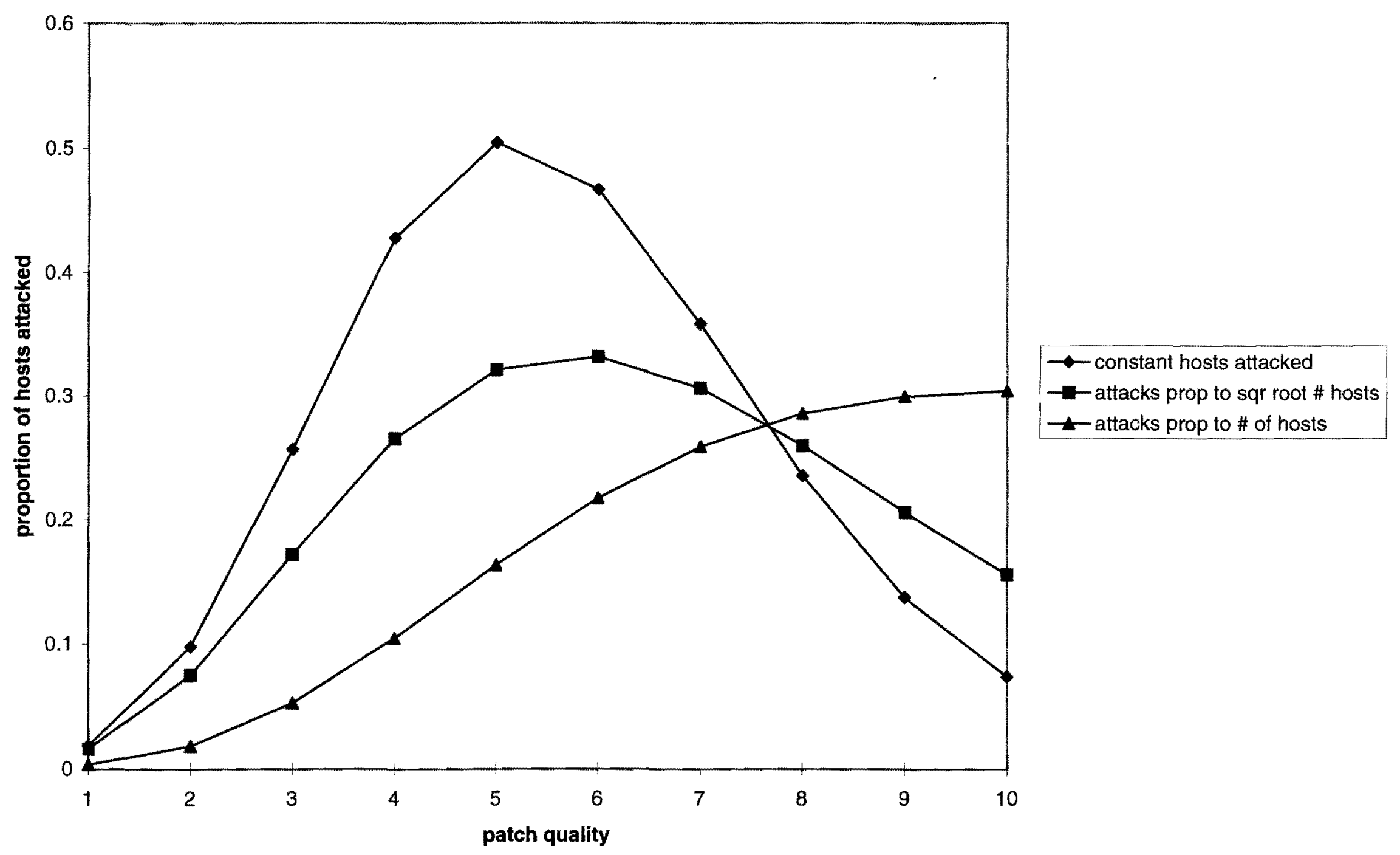


Figure 4b. Proportion of hosts attacked in a so-so environment

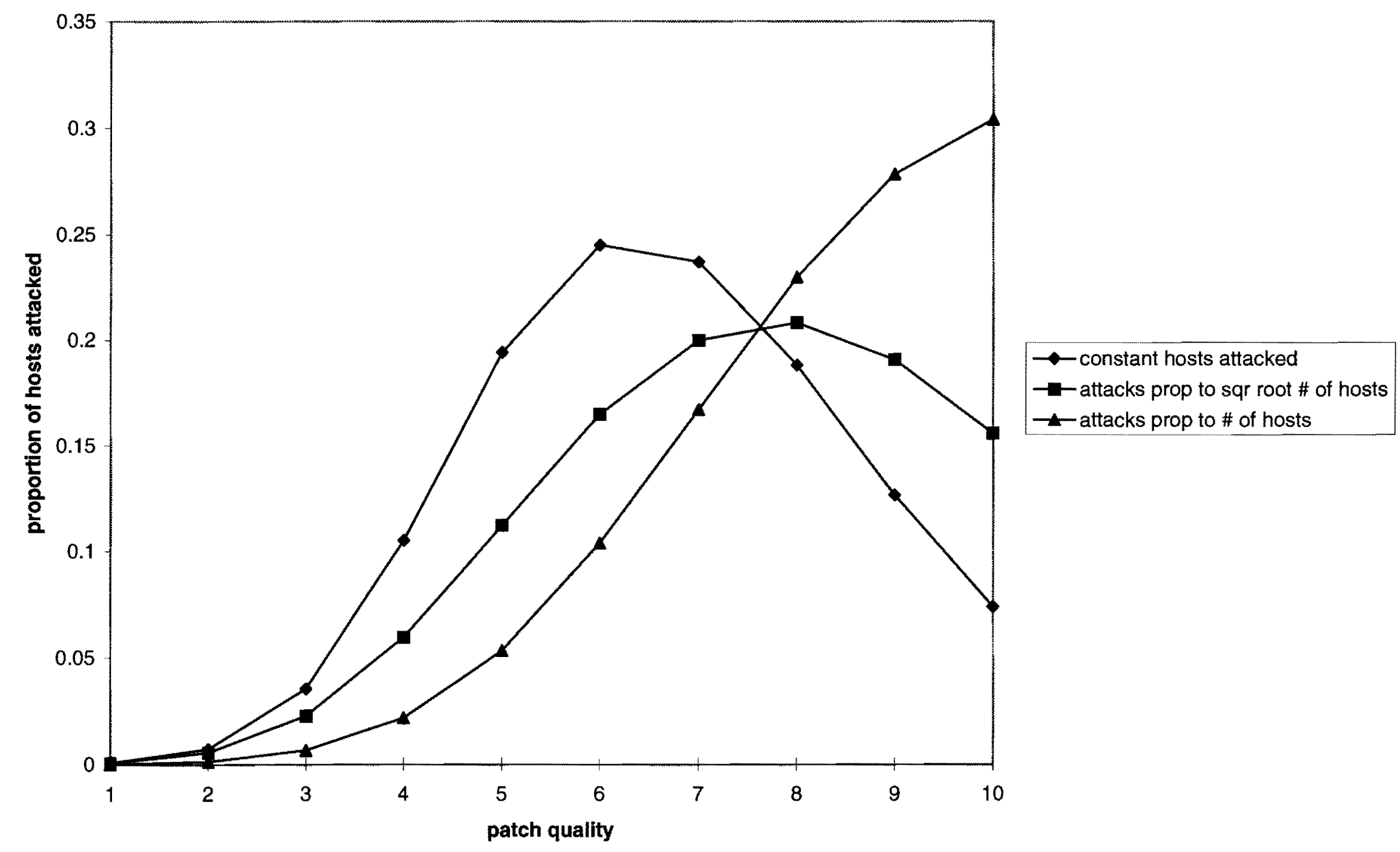


Figure 4c. Proportion of hossts attacked in a good environment

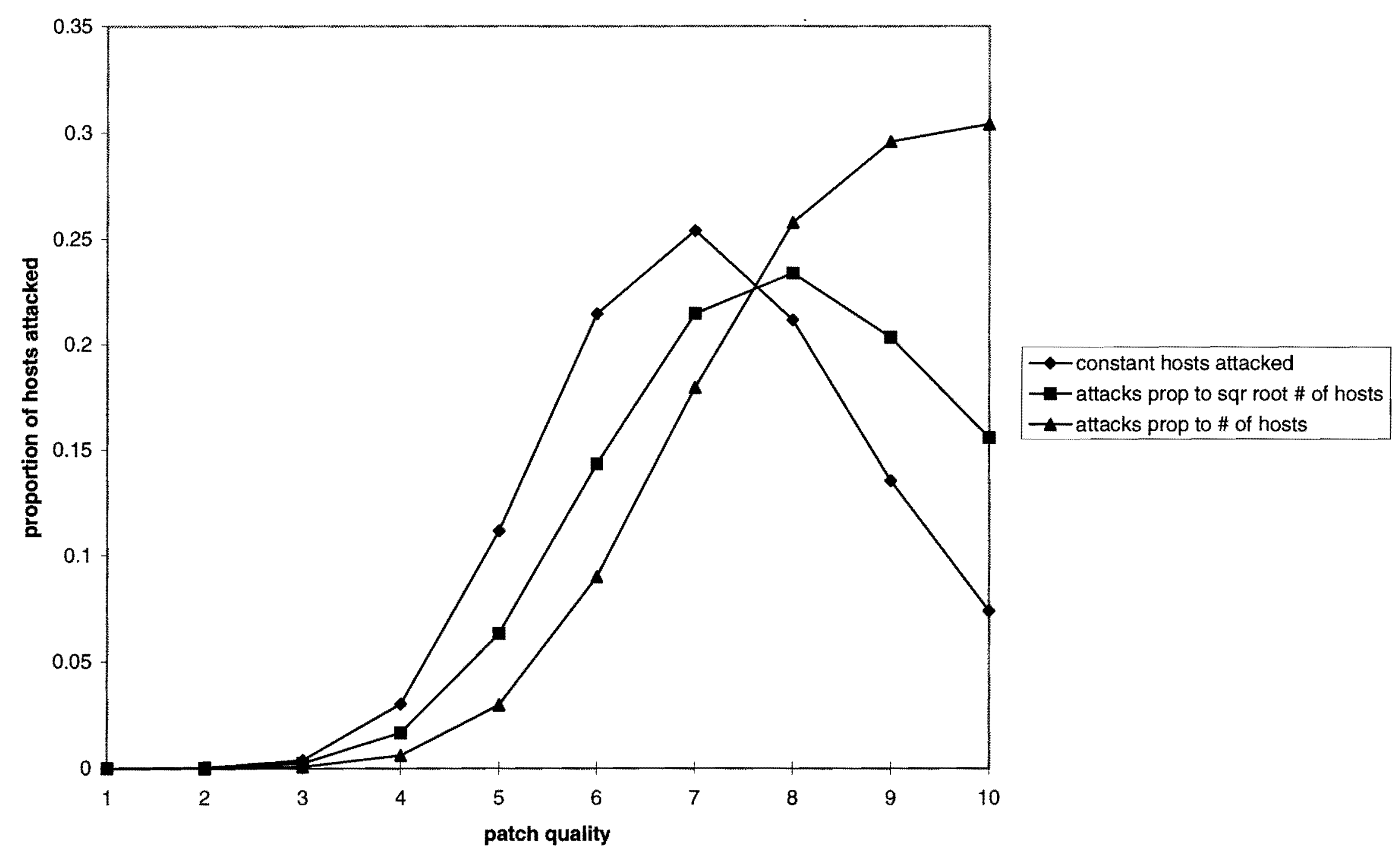




\section{Annotated TURBO BASIC program to calculate}

a) limiting opinion distribution,

b) willingness to accept patch types, and

c) pattern of exploiting patches.

The distribution of patch types, $f(x)=f(i)$, the numbers of hosts in each patch type, $M(x)=m(i)$, and the numbers of hosts attacked, $A(x)=a 1(i)$ [constant proportion attacked], or a2(i) [attacks proportional to the square root of the number of hosts], or a3(i) [attacks proportional to the number of hosts] are calculated in lines 100 to 135 .

The probabilities of encountering a patch worse than type i [called l(i)], or better than type $\mathrm{i}$ [called $\mathrm{r}(\mathrm{i})$ ] are calculated in lines 140 to 190 and 240 to 290 , respectively. These probabilities are used in analyzing the opinion-change rule.

After initializing [opinion probabilities, $\mathrm{g}(\mathrm{i})$, are set $=0$ in lines 500 to 530 and transitions probabilities $p(i, j)$, are set $=0$ in lines 600 to 640$]$, transition probabilities are calculated for the four rules for opinion change:

(i) "one step up and one step down" in lines 700 to 780 ,

(ii) "one step up and all the way down" in lines 805 to 970 ,

(iii) "all the way up and one step down" in lines 1020 to 1170 , and

(iv) "all the way up and all the way down" in lines 1205 to 1240 .

The Markov program for opinion change is run for 100 time steps in lines 1500 to 1690 , resulting in the limiting distribution of opinions, $g(j)$, for patch types $i$.

Willingness to accept patches is calculated in lines 1700 to 1770 .

Finally, the expected proportions of hosts attacked in each patch type for three difference exploitation rules are calculated in lines 2500 to 2540 . The numbers of patch types, $n=10$, is given in line 10 , and the average number of parasitoid visits per patch, $b=2$, is given in line 20 . 


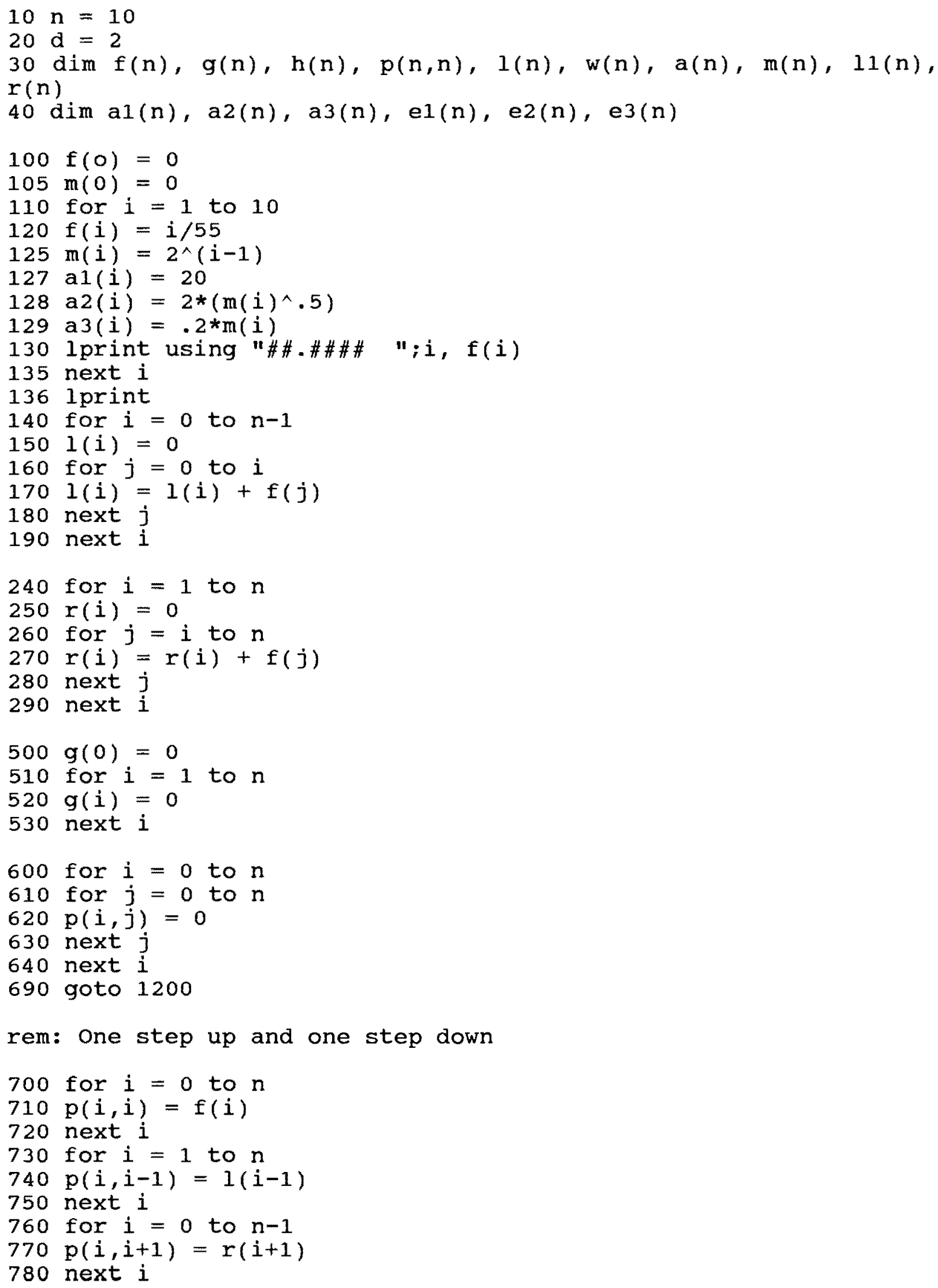




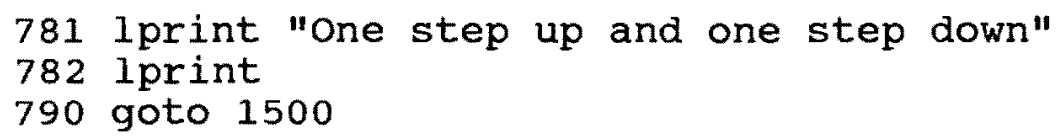

rem: All the way up and one step down

1000 lprint "All the way up and one step down" 1010 lprint

rem: All the way up and all the way down

1200 lprint "All the way up and all the way down" 1201 lprint

1205 for $i=0$ to $n$

1210 for $j=0$ to $n$

$1220 p(i, j)=f(j)$

1230 next j

1240 next $i$ 


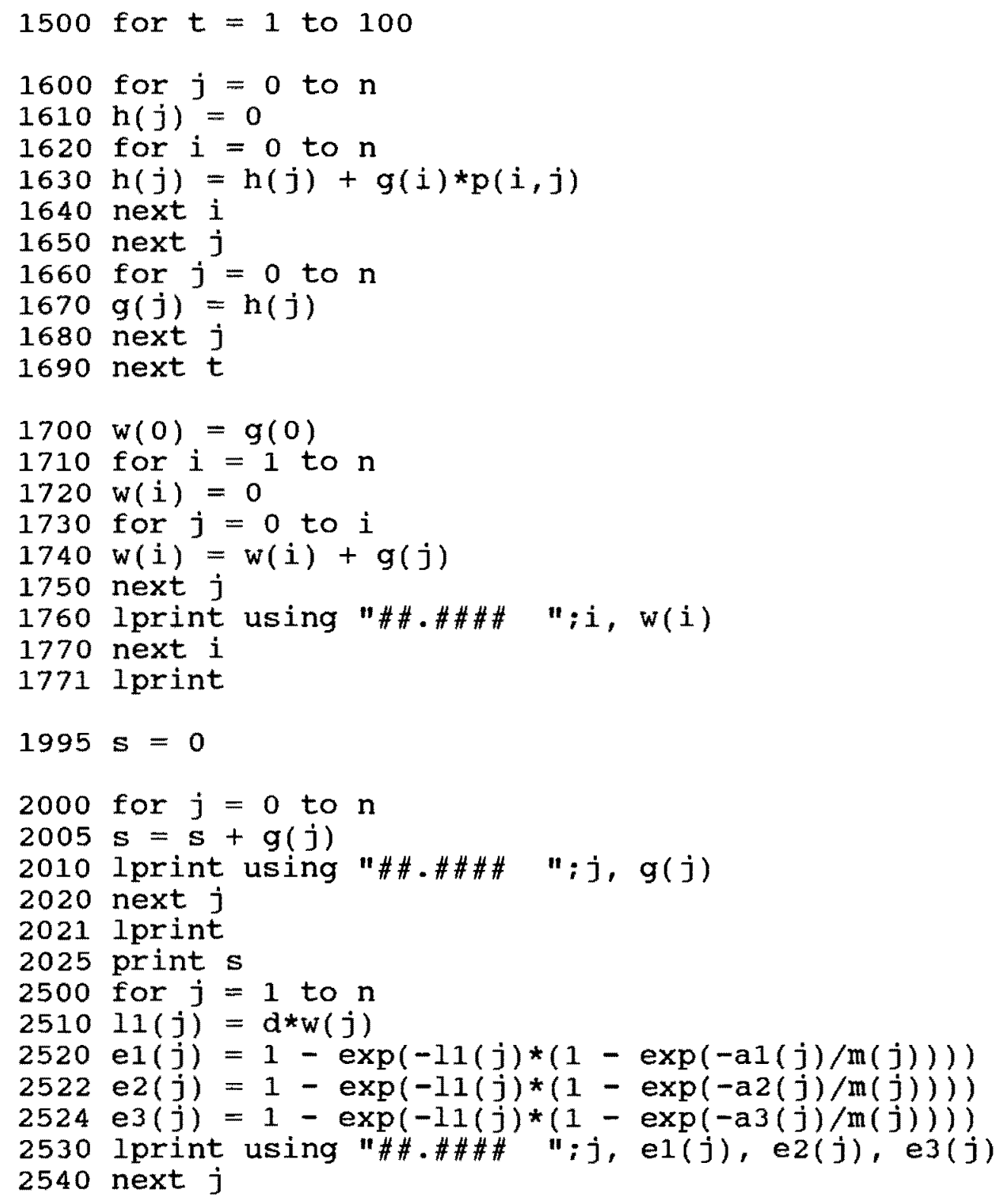



1.0000
0.0182
2.0000
0.0364
3.0000
0.0545
4.0000
0.0727
5.0000
0.0909
6.0000
0.1091
7.0000
0.1273
8.0000
0.1455
9.0000
0.1636
10.0000
0.1818

All the way up and all the way down
1.0000
0.0182
2.0000
0.0545
3.0000
0.1091
4.0000
0.1818
5.0000
0.2727
6.0000
0.3818
7.0000
0.5091
$8.0000 \quad 0.6545$
$9.0000 \quad 0.8182$
$10.0000 \quad 1.0000$
0.0000
0.0000
1.0000
0.0182
2.0000
0.0364
3.0000
0.0545
$4.0000 \quad 0.0727$
$5.0000 \quad 0.0909$
$6.0000 \quad 0.1091$
$7.0000 \quad 0.1273$
$8.0000 \quad 0.1455$
$9.0000 \quad 0.1636$
$10.0000 \quad 0.1818$
1.0000
0.0357
2.0000
0.1033
0.0310
0.0066
0.0793
0.0196
0.1948
0.1288
0.0388
4.0000
0.2838
0.1683
0.0638
5.0000
0.3224
0.1932
0.0941
6.0000
0.2988
0.2034
0.1293
7.0000
0.2017
0.1685
0.2391
0.1911
0.2112
8.0000
0.1725
0.1157
0.1749
0.0738
0.2567
10.0000
0.1557
0.3041 